\title{
Potencial de aprovechamiento de la biomasa vegetal como aislamiento en climas extremos del Ecuador
}

\section{(Potential use of vegetal Biomass as insulation in extreme climates of Ecuador)}

\author{
Luis Velasco Roldan ${ }^{1}$, Leonardo Goyos Pérez ${ }^{1}$, Luis Freire Amores ${ }^{2}$, Alexander lbarra ${ }^{1}$
}

\section{Resumen:}

Un factor de gran riqueza ecológica de Ecuador está vinculado a su tradición en el uso de fibras y otros residuos de carácter orgánico. Estos materiales compuestos se emplean como elemento de relleno, refuerzo o aislamiento térmico en el campo de la construcción de viviendas, lo que conlleva un gran potencial en el marco de la arquitectura más sustentable. En Ecuador, la variabilidad climática y el bajo poder adquisitivo de muchos de sus habitantes obliga a utilizar materiales de construcción de presencia local y bajo o nulo costo, con el fin de lograr una vivienda económica y confortable. Es por ello que se han analizado la presencia de recursos o desechos naturales de biomasa a nivel regional confrontándolos la tradición constructiva, para posteriormente superponerlos geográficamente con las principales variables climáticas que afectan a la eficiencia energética. De esta forma es posible determinar qué, dónde y cómo utilizar los distintos recursos la biomasa para permitir una respuesta edificatoria que cuente con una sólida argumentación social, económica, medioambiental y energética con el objetivo de facilitar las condiciones adecuadas para el acceso a un hábitat económico, seguro, eficiente y digno.

Palabras clave: residuos agrícolas, aislamiento térmico, eficiencia energética, recursos naturales.

\begin{abstract}
:
In Ecuador, a factor of great ecological wealth is linked to its tradition in the use of fibers and other organic waste composite character as filler element, reinforcement or insulation in the field of housing construction, which carries great potential under the most viable architecture. The climate variability in Ecuador and the low purchasing power of their inhabitants forced to use local available building materials inexpensive or at no cost, in order to achieve economic and comfortable housing. That is why we have analyzed the presence of natural resources and waste biomass confronting regional building tradition, later superimpose geographically with major climatic variables affecting energy efficiency. This makes it possible to determine what, where and how to use the different biomass resources to allow a response to build that has a strong social, economic, environmental and energy argument in order to facilitate the conditions for access to economic habitat efficient, safe and dignified.
\end{abstract}

Keywords: agricultural waste, thermal insulation, energy efficiency, natural resources.

\footnotetext{
${ }^{1}$ Universidad de las Fuerzas Armadas, Sangolquí - Ecuador ( luisvelascoroldan@espe.edu.ec )

${ }^{2}$ Universidad Tecnológica Equinoccial, Quito - Ecuador ( luis.freire@ute.edu.ec )
} 


\section{Introducción}

El desarrollo de aislantes térmicos basados en residuos de biomasa es una línea de investigación prometedora que permitirá la mejora sustancial de la eficiencia energética y el confort térmico del hábitat en el ámbito ecuatoriano con soluciones sustentables de bajo coste. Existen múltiples componentes edificatorios ancestrales como muros o pisos susceptibles de evolución y mejora mediante la adición de altos porcentajes de biomasa en su composición. Por otro lado, el desarrollo de elementos constructivos contemporáneos como paneles o mantas basados en materiales vegetales permitirá la mejora de la sustentabilidad de soluciones industriales así como la reducción importante del coste de producción de estas.

Uno de los principales obstáculos para el desarrollo de dichas técnicas constructivas es el reducido conocimiento de los recursos de biomasa disponibles en el país, así como el potencial aislante de estos en sus distintas posibilidades de aglomeración.

Investigaciones en torno al uso de residuos vegetales, han demostrado su capacidad de mejorar la relación resistencia/densidad de los hormigones a la vez que reducir los agregados pétreos de este. (Beraldo, 2011. Dicker, 2014. Juárez, 2010. Katzer, 2013. Mantia, 2011). La restricción fundamental en estos casos resulta la disminución de resistencia que induce la presencia de los espacios de aire de la biomasa. No obstante algunas aplicaciones que requieren la resistencia a esfuerzos de flexión se verían favorecidas con la aplicación de fibras para la fabricación del elemento. Ciertas especies vegetales presentan un sistema vascular que atrapa el aire en su interior al desecarse. Esta notable proporción de aire permitirá un efecto aislante y la reducción de áridos. Teniendo en cuenta que el coeficiente de conductividad se reduce proporcionalmente al aumento de la biomasa y la resistencia disminuye de forma invérsamente proporcional, el porcentaje de biomasa posible a usar estará limitado por la resistencia necesaria a garantizar.

En torno a dicho efecto se encuentran estudios de ciertas biomasas, como la cascarilla de arroz o café, en torno al potencial que atesoran como aislamiento térmico conductivo de bajo coste (Salazar, García, Olaya, n.d., Serrano,Castro,1985). Pese a ello, no se han realizado aún estudios en profundidad en torno al potencial aislante de las posibles biomasas aprovechables en Ecuador.

Compuestos encaminados a determinar las posibilidades de inclusión de los residuos de biomasa generados por los cultivos propios de cada zona en elementos constructivos tales como: bloques, adobes, tapial o pisos de tierra estabilizados, paneles, mantas, etc. (Velasco, Delgado, Goyos, n.d.) se encuentran en fase final de evaluación por parte del Grupo de Energías Renovables del Departamento de Ingeniería Mecánica de la Escuela Politécnica del Ejército con resultados muy prometedores por sus bajas conductividades. Los ensayos de conductividad están siendo realizados mediante prototipo desarrollado por el equipo redactor para la determinación de la conductividad térmica de las biomasas de estudio según las normas de la ASTM 177-13 (Method, 
2013). Dicha norma establece criterios de laboratorio para la medida del flujo de calor en estado estacionario a través de una muestra plana y homogénea de caras paralelas monitoreada a temperaturas constantes mediante un sistema llamado de placa caliente. (Velasco, L., Delgado, R., \& Goyos, L., Freire (2015) . Los resultados preliminares se resumen en la Tabla 1.

Tabla 1. Conductividades de las distintas biomasas ensayadas

\begin{tabular}{|c|c|c|}
\hline Material & Densidad Aparente $\left.\mathbf{( g r} / \mathbf{c m}^{\mathbf{3}}\right)$ & Coeficiente $\left(\mathbf{W} / \mathbf{o K}_{\mathbf{m}} \mathbf{}^{\mathbf{2}}\right)$ \\
\hline Raquis Palma a granel & 0,182496 & 0,085936106 \\
\hline Raquis Palma a granel & 0,148278 & 0,061027338 \\
\hline Bagazo de caña de azucar & $0,062222-0,078493$ & $0,025505969-0,03407669$ \\
\hline Cascarilla de Cacao & 0,078889 & 0,021480109 \\
\hline Virutas Guadua. Sección 2-3 cm & 0,078493 & 0,03886449 \\
\hline Totora granel. Longitud: $1-2 \mathrm{~cm}$ & 0,078493 & 0,032098209 \\
\hline
\end{tabular}

La distribución de las estaciones meteorológicas en Ecuador así como la relativa escasez de datos climatológicos dificulta la planificación de soluciones constructivas adaptadas al clima con la requerida precisión geográfica. Dada la falta de datos reales climáticos en Ecuador, el desarrollo del análisis climático se realiza además de mediante el estudio de fuentes bibliográficas (Cañadas, 1983) y de los datos reales procedentes de las estaciones meteorológicas entre los años 2004 y 2014 (INAMHI, n.d.) se han incluido modelizaciones climáticas allá donde los datos reales son insuficientes ("climate.data.org," n.d.)

\section{Desarrollo}

El desarrollo de la investigación pretende por lo tanto, determinar las zonas con condiciones climáticas que requieran sistemas constructivos dotados de aislamiento térmico, determinar las biomasas potencialmente utilizables como aislamiento térmico en el entorno local y sugerir los posibles usos de esta biomasa.

\subsection{Biomasas potencialmente utilizables}

De acuerdo con las necesidades de los diferentes elementos presentes en la edificación y las posibilidades de uso de las diferentes biomasas, éstas pueden ser clasificadas en dos grandes grupos: la biomasa que presenta fibras largas y constituyen un interesante refuerzo para elementos cargados a tracción y flexión y la biomasa particulada o con fibras cortas que sólo son interesantes en aplicaciones de aislamiento térmico en materiales de construcción en masa ligados mediente un aglomeráte, en general tierra o cemento (Salazar, 2007). En ambos casos el aporte de biomasa al elemento redunda en un aumento de la capacidad aislante y de la ligereza final del material. Las biomasas potencialmente utilizables pueden caracterizarse en función del hábitat característico de cada especie, su importancia económica y su forma de uso tradicional. Esta última característica resulta la de mayor interés para nuestros fines. 


\subsubsection{Especies de utilidad económica que producen un desecho vegetal}

Son especies para uso como alimento humano o animal y que producen un desecho con potencial aprovechamiento para materiales compuestos. En Ecuador es notable el caso del raquis de plátano o la palma africana , la tusa de maiz, el bagazo de caña de azúcar, las cascarillas de café, cacao o arroz y las diferentes fibras de palmeras como el coco, etc. (IPN, 2007, Alvarez-Castillo, 2013, Ali, Liu, Chouw, 2012, Biagiotti, Puglia, Kenny, 2004)

La mayor limitación en el uso de estas especies radica en su aplicación en otros usos de los ya implantados: como las hojas de palmera para techumbre, como abono orgánico, forraje o para la producción de energía mediante su quema.

\subsubsection{Especies que son en sí mismas de utilidad económica}

Este grupo lo constituyen las fibras textiles y las plantas usadas comúnmente como cordelería o tejidos como es el caso de los agaves, el algodón, la cabuya, el fique y el abacá o cáñamo de Manila por citar los más usados. En este grupo se pueden incluir también especies de aplicación en producciones artesanales y en ocasiones, en la construcción: especies como la totora, la caña, la caña brava, la guadua o bambú, la toquilla, los juncos y los mimbres. Estarían también incluidas en este grupo las numerosas especies maderables con aplicaciones especiales como el caucho y los ficus gomosos. Las especies tubulares como las cañas también han sido y son usadas como elementos constructivos en sí mismos, como es el caso del bambu, la caña y la totora. A pesar de ello existe un potencial importante en el uso de sus fibras para compuestos tanto poliméricos como cerámicos o como elementos aislantes en techo (Eguiguren, 2013, Romero, Weeks, Huelman, 2012, Izco, 2007).

La mayoría de las especies productoras de hilos y cuerdas como la cabuya, el penco o el ágabe, tienen su aplicación bien determinada, tienen poca producción excedente y no resulta económicamente rentable su uso en viviendas. A pesar de ello, en algunos casos con excedente de producción sería factible su aplicación en la restitución de capacidad resistente en adobes o tapiales en unión de otras biomasas aislantes de fibra corta o en el refuerzo de paneles aglomerados de biomasa.

En el caso de las especies maderables, generalmente resulta más rentable usar las mismas como tabla que utilizar sus fibras. Pese a ello, existen posibilidades en el uso de las fibras procedentes de ramas jóvenes (ficus, guadua, etc.) o de residuos procedentes de su manufactura (serrin, astillas, etc.). Teniendo en cuenta la experiencia ancestral en el uso de algunas especies autóctonas, el enfoque de este grupo de especies se centrará en el aprovechamiento de partes no totalmente aprovechadas o aplicaciones no comunes. 


\subsubsection{Especies sin utilidad económica aparente}

Este grupo resulta de especial interés precisamente por no tener una utilidad económica clara. Especies como el pajonal (Izco, 2007, De la Torre, 2008), se han usado tradicionalmente para reforzar los adobes. El incremento del porcentaje de pajonal en compuestos de tierra se presenta como un prometedor recurso aislante de bajo coste siempre y cuando exista un control para una explotación estríctamente local y sustentable.

La Tabla 2 resume el estudio de la localización regional de las distintas biomasas detectadas con posibilidades de aprovechamiento aislante, determinando además su hábitat, su parte aprovechable, así como sus usos actuales en la construcción.

Tabla 2. Biomasas presentes en Ecuador y sus principales características.

\begin{tabular}{|c|c|c|c|c|}
\hline $\begin{array}{c}\text { Nombre } \\
\text { común }\end{array}$ & $\begin{array}{c}\text { Nombre } \\
\text { científico }\end{array}$ & Hábitat & Parte aprovechable & Uso actual en la construcción \\
\hline \multicolumn{5}{|l|}{ LEÑOSOS } \\
\hline $\begin{array}{c}\text { Caña } \\
\text { Guadúa } \\
\end{array}$ & Bambusa guadua & Clima Tropical & Tallo (fibra larga, piezas) & $\begin{array}{c}\text { Estructura, cerramientos, } \\
\text { andamios, encofrados, esterillas }\end{array}$ \\
\hline Guadua & $\begin{array}{c}\text { Guadua } \\
\text { angustifolia }\end{array}$ & Bosque húmedo tropical, márgenes de ríos, preferible a alturas $<200 \mathrm{~m}$ & Tallo (fibra larga, piezas) & $\begin{array}{c}\text { Estructura, cerramientos, } \\
\text { andamios, encofrados, esterillas }\end{array}$ \\
\hline Carrizo & Arundo donax & Duna arenosa ,zonas húmedas, borde de ríos entre 0 y $4000 \mathrm{~m}$. & Tallo (fibra larga) & Esterillas y decorativos \\
\hline Higuerón & $\begin{array}{c}\text { Ficus } \\
\text { luschnathiana } \\
\end{array}$ & Clima tropical e intertropical & $\begin{array}{c}\text { Guías jóvenes (fibra larga, } \\
\text { lianas) }\end{array}$ & Sogas \\
\hline Caucho & $\begin{array}{l}\text { Castilla elástica, } \\
\text { Hevea brasiliensis }\end{array}$ & Clima tropical e intertropical & $\begin{array}{l}\text { Guías jóvenes (fibra larga, } \\
\text { lianas) }\end{array}$ & Sogas \\
\hline Matapalo & $\begin{array}{c}\text { Ficus } \\
\text { nymphaeifollia } \\
\end{array}$ & Clima tropical e intertropical & $\begin{array}{c}\text { Guías jóvenes (fibra larga, } \\
\text { lianas) }\end{array}$ & Sogas, caucho natural \\
\hline Balsa & $\begin{array}{c}\text { Ochroma } \\
\text { pyramidale }\end{array}$ & Clima tropical, bosques $30-400 \mathrm{~m}$ & Corteza (fibra larga) & Sogas \\
\hline Ceibo & $\begin{array}{l}\text { Erythrina } \\
\text { cristagalli }\end{array}$ & Bosque seco tropical & $\begin{array}{c}\text { Corteza, madera (piezas, } \\
\text { fibra larga }\end{array}$ & Madera \\
\hline \multicolumn{5}{|l|}{ MIMBRES } \\
\hline Totora & $\begin{array}{l}\text { Schoenoplectus } \\
\text { californicus }\end{array}$ & Humedales, , zona lacustre andina, tierras salinas y empantanadas & Tallo (fibra larga, piezas) & Techumbres \\
\hline Totorilla & Juncus arcticus & Humedales, zona lacustre andina & Tallo (fibra larga, piezas) & - \\
\hline Sigse & $\begin{array}{c}\text { Cortaderia nitida } \\
\text { (kunth) Pilg. }\end{array}$ & Clima de alta montaña paramos, tierras altas entre 3000 y $4500 \mathrm{msnm}$ & Tallo (fibra larga) & Techumbres \\
\hline \multicolumn{5}{|l|}{ TEXTILES } \\
\hline Abacá & Musa textilis & Clima cálido lluvioso & Tallo (fibra larga) & Sogas \\
\hline Cabuya & Agave sisalana & Clima árido. Terrenos pedregosos, arenosos y de baja productividad agricola & Hojas (fibra larga) & Sogas, sacos \\
\hline $\begin{array}{l}\text { Ágave } \\
\text {,Penco } \\
\end{array}$ & Agave americana & Clima árido. Terrenos pedregosos, arenosos y de baja productividad agrícola & Hojas (fibra larga) & Sogas, sacos \\
\hline \multicolumn{5}{|c|}{ PALMERAS } \\
\hline Toquilla & $\begin{array}{l}\text { Carludovica } \\
\text { palmata }\end{array}$ & Clima tropical & Tallo (fibras, cintas) & - \\
\hline Coco & Cocos nucifera & Clima cálido húmedo & $\begin{array}{l}\text { Fruto, palmas, madera (fibras, } \\
\text { piezas) }\end{array}$ & - \\
\hline Mocora & $\begin{array}{l}\text { Astrocaryum } \\
\text { standleyanum }\end{array}$ & Clima tropical hasta $500 \mathrm{~m}$ & $\begin{array}{c}\text { Tallo, hojas (tablas, cinta, } \\
\text { fibras) }\end{array}$ & - \\
\hline Pambil & Iriartea deltoidea & Clima Tropical & Tablas & Estructura, techumbres \\
\hline Bejuco real & Cissus verticillata & Clima tropical & Tallo & Sogas \\
\hline \multicolumn{5}{|l|}{ UTILES } \\
\hline Maíz & Zea mays & Tropical, subtropical, frio & Tusa o mazorca & - \\
\hline Café & $\begin{array}{c}\text { Coffea arábica, } \\
\text { Coffea canephora }\end{array}$ & Zonas altas $<500-1700$, no mas de $3000 \mathrm{~mm}$ lluvia anual & $\begin{array}{c}\text { Cascarilla (seca) } \\
\text { pulpa y cáscara (húmeda) }\end{array}$ & - \\
\hline Cacao & Theobroma cacao & Bosque tropical. Requiere sombra y humedades moderadas & Cascarilla y cáscara & - \\
\hline Arroz & Oryza sativa & Clima tropical. humedales, & Cáscarilla & - \\
\hline $\begin{array}{l}\text { Caña de } \\
\text { azúcar }\end{array}$ & $\begin{array}{l}\text { Saccharum } \\
\text { officinarum }\end{array}$ & Clima tropical, subtropical & Bagazo de caña de azucar & - \\
\hline Banano & Musa paradisiaca & Clima tropical húmedo & Raquis, tallo, hojas & - \\
\hline
\end{tabular}




\subsection{Distribución geográfica de la biomasa}

Determinar con exactitud la distribución geográfica de las distintas especies resulta difícil. Resultan de gran ayuda algunas obras sobre el tema pero existen especies con presencia escasa en micro-localizaciones específicas pero en todas las provincias del país. (Erriguren, 2013, Romero, Izco, 2007, De la Torre, 2006, INEC, 2009, Macía, 2006)

Una propuesta de uso de biomasas en función de su distribución local debe forzosamente centrarse en aquellas de fácil acceso, por medio de su presencia natural, por necesidades de la distribución alimentaria o por su coste nulo. Desde este punto de vista es posible entonces centrar la atención en aquellas especies que cumplen con estas condicionales.

\subsection{Análisis de las condiciones climáticas}

Con el objetivo de situar geográficamente los requerimientos exigibles al hábitat y confrontarlos con las biomasas disponibles, se han representado sobre los mapas de las figuras 1 y 2 , además de los climas (INAMHI, n.d.), las variables climáticas que puedan condicionar la estrategia constructiva a seguir: temperatura (medias de las mínimas y medias de las máximas anuales), humedad relativa (media de las máximas y media de las mínimas anuales), nubosidad, radiación y presencia de viento.

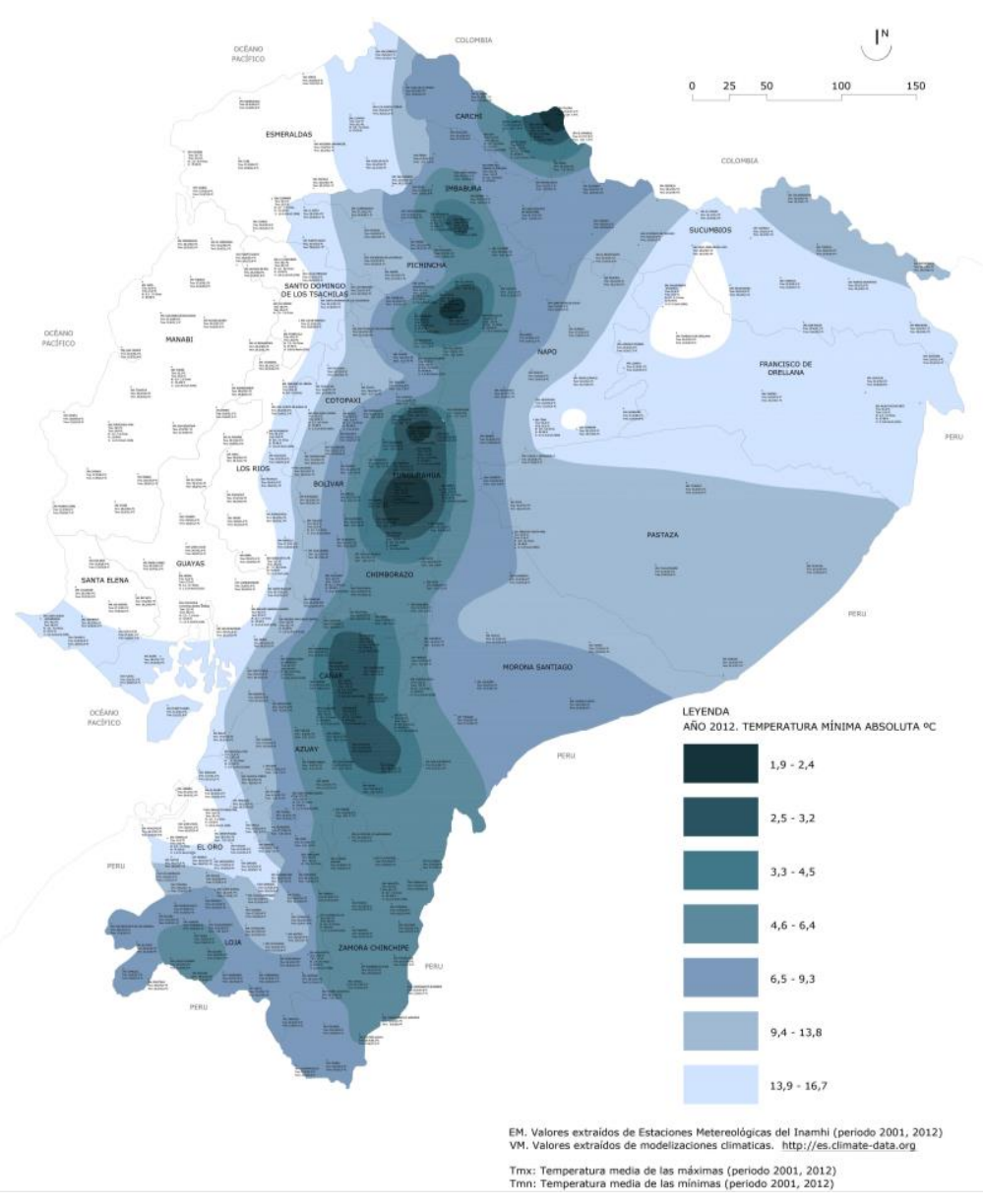

Figura 1. Mapa de temperaturas mínimas (absolutas y medias) 
Del estudio de los datos y bibliografía existentes puede determinarse que, pese a que climáticamente en Ecuador se considera que existe una época lluviosa y otra temporada seca, la estabilidad térmica en términos anuales es importante, no siendo así en términos geográficos, en donde la variabilidad es extrema, si bien existen patrones comunes en grandes zonas climáticas: Costa, Sierra y Amazonia (Pourrut, Rovere, Romo, \& Villacrés, 1995). Dichas zonas corresponden a grandes rasgos con una biodiversidad que ha determinado, junto con la cultura de sus pobladores unos patrones edificatorios cuasi comunes que nos permiten estructura el análisis en tres grandes zonas.

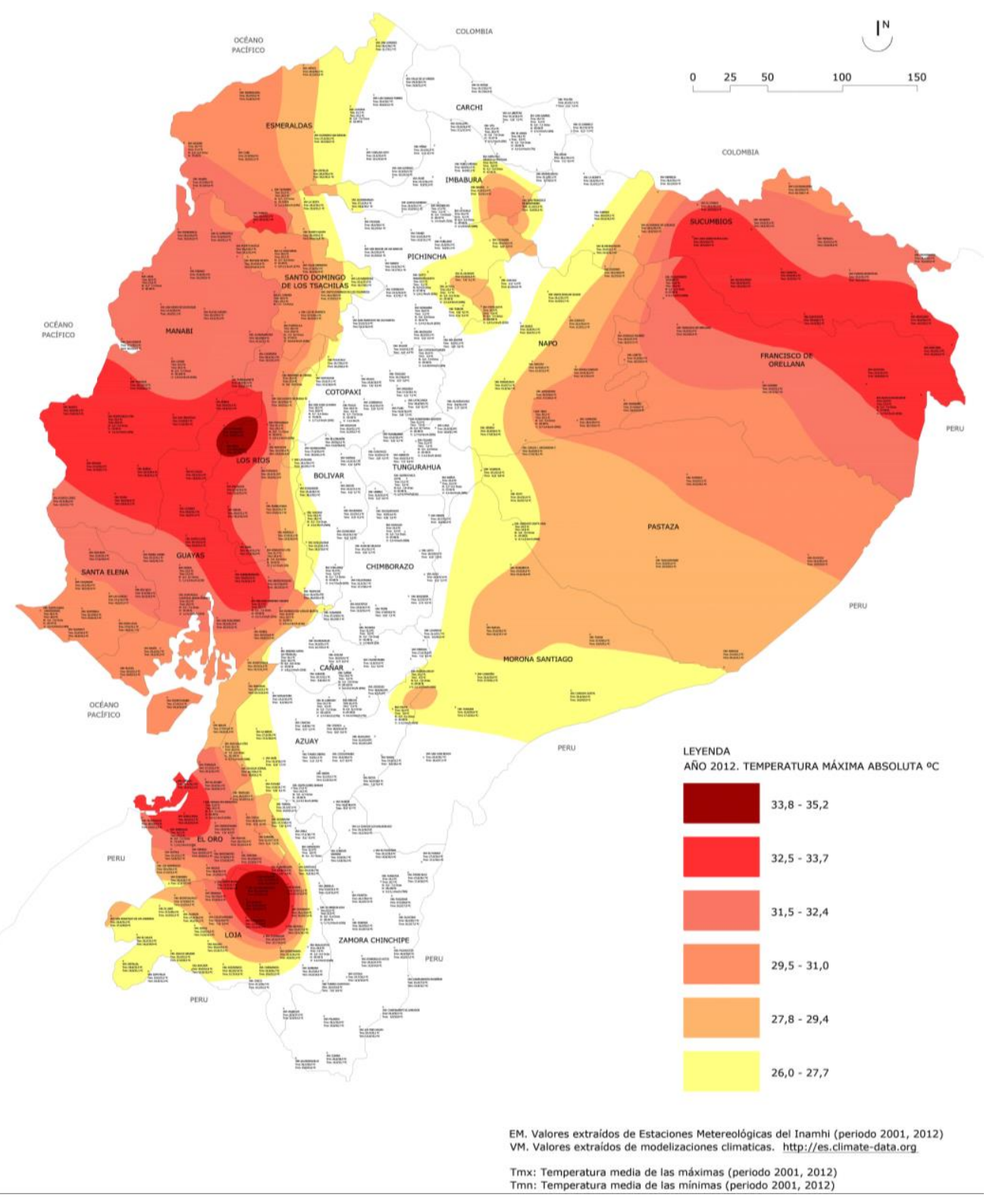

Figura 2 . Mapa de temperaturas máximas (absolutas y medias) 


\subsection{Variantes constructiva tradicionales y usos propuestos de las biomasas existentes en función del clima}

La investigación en torno al utilización potencial de utilización de la biomasa como aislante en elementos de construcción se centra en el uso en cerramiento (paredes y pisos principalmente), y los elementos de revestimiento y relleno (Velasco, Delgado, Goyos, n.d.). En el caso de los cerramientos, el mayor interés radica en la sustitución de áridos y en menor grado de cemento, yeso o arcilla, buscando con ello una mayor economía de recursos económicos y ambientales , a la vez que se aporta una mejora considerable en su capacidad aislante. En el caso los elementos no portantes de revestimiento o relleno como paneles para falso techo (cielo raso), relleno de cámaras de paredes o techos, etc, el objetivo será determinar como aglomerar la fibra de la forma menos contaminante y como preservarla del ataque de mohos o insectos. Puede estudiarse en detalle las distintas lineas de investigación abiertas en torno al tema en el artículo: Investigación y desarrollo de aislantes térmicos naturales basados en residuos de biomasa para su aplicación en la mejora de la eficiencia energética de las edificaciones en América Latina (Velasco, Delgado, Goyos, n.d.).

\subsubsection{Región Costa}

La región geográfica de costa ecuatoriana comprende las provincias de Esmeraldas, Guayas, Los Ríos, Manabí, Santa Elena y El Oro, pese a que pueden considerarse de esta, desde el punto de vista climático, la zona más costera de las provincias de Cañar, Azuay y Santo Domingo. La región litoral es seca y árida con continuas corrientes de aire procedentes del mar que atemperan las temperaturas máximas y mínimas, existiendo una diferencia máxima anual de temperaturas de $3^{\circ} \mathrm{C}$ y una diferencia térmica diaria máxima de $7^{\circ} \mathrm{C}$. Las temperaturas medias oscilan entre los 20,5 y 30,5 C. La humedad relativa varía diariamente entre el 55 y el 100\%, manteniéndose relativamente estable durante todo el año. El interior es muy cálido y húmedo y comprende los territorios de la costa interna hasta los declives de la cordillera Occidental. Las temperaturas medias de las máximas oscilan entre los 18 y los $32^{\circ} \mathrm{C}$ a lo largo del año. La humedad relativa es alta: entre el 70 y el $100 \%$. Se caracteriza por las constantes precipitaciones debido al efecto de las corrientes marinas de Humboldt y el Niño (AEPEREACU, n.d.). La radiación en toda la región de la costa es intensa: entre los 3,5 y 4 y $4,5 \mathrm{Kwh} / \mathrm{m}^{2}$. 

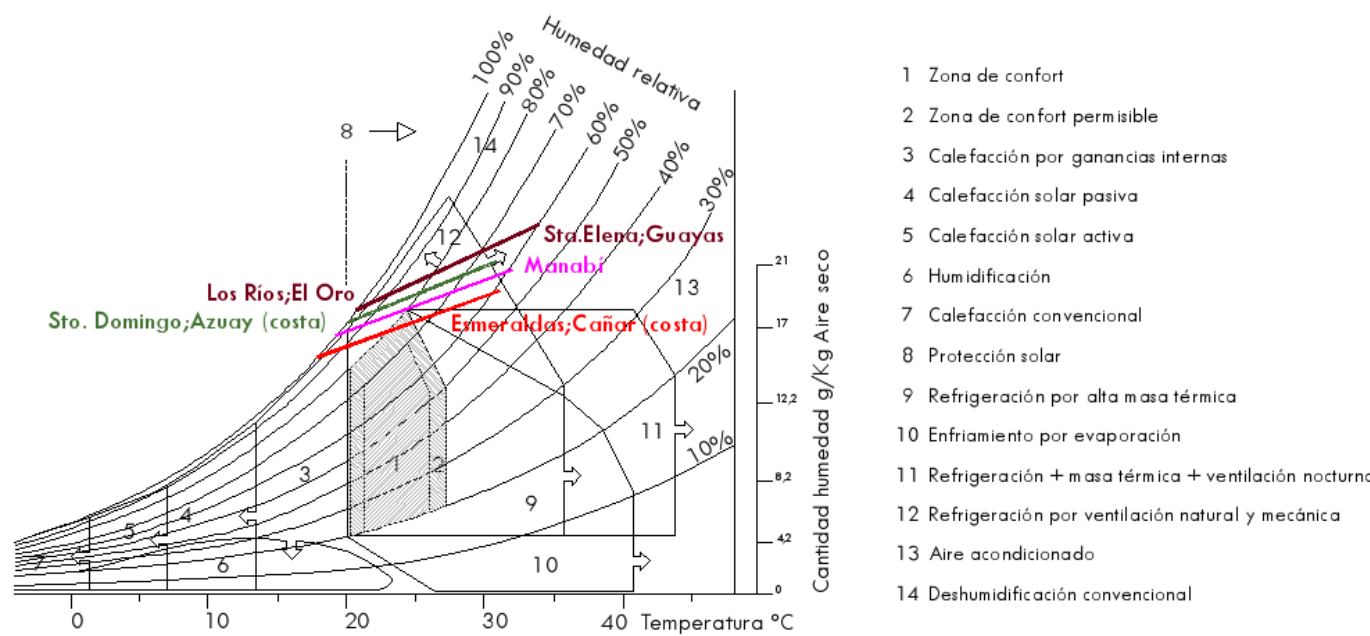

Figura 3. Gráfico bioclimático. Provincias de la región climática de la costa.

En la Figura 3 se observa como el principal problema climático de la región (especialmente el las provincias del sur) son las altas temperaturas (principalmente en horas centrales del día), asociadas a altos niveles de radiación, así como frecuentes porcentajes de humedad relativa superiores al $80 \%$ (incrementándose hacia la zona norte). No hay en la costa problemas de confort por bajas temperaturas. Por el contrario, al no descender la temperatura durante la noche no es posible la refrigeración por ventilación nocturna. Se observa que en toda la zona es siempre necesaria la protección solar.

La construcción tradicional en la costa presenta una perfecta adaptación al clima de una forma sostenible gracias a la limitada huella de carbono de sus materiales, a la ausencia de inercia térmica y la absoluta permeabilidad de toda la envolvente que facilita la refrigeración y evita la acumulación de humedad sin necesidad de ventanas. Los suelos y las paredes están construidos de pambil, guadua o caña picada sobre latilla horizontal o vertical de guasango o laurel. Se encuentra generalmente levantada del suelo entre 60 y $150 \mathrm{~cm}$. con postes de chonta o guadua. El techo es elaborado de rampira o bijao (hojas de palma o de plátano), y generalmente se prolonga más allá de los cerramientos verticales para dotar a estos de protección frente a la radiación formando los corredores y espacios exteriores protegidos del sol, hacia los que se vuelcan diversas actividades de la casa. (Nurnberg, Estrada, 1982). Presenta problemas puntuales de seguridad y mantenimiento debido a la degradación de las fibras vegetales por pudrición o ataque de insectos.

La sustitución de los tipos constructivos tradicionales por sistemas constructivos basados, casi invariablemente, en muros de bloques de hormigón y cubiertas de fibrocemento o chapa y losas de hormigón supone un empeoramiento radical de la sostenibilidad edificatoria al utilizar materiales no reciclables, procedentes de zonas lejanas y basados en el hormigón o el acero. La eficiencia energética de la vivienda, lejos de mejorarse, empeora radicalmente al reducir la ventilación en las edificaciones impidiendo la disipación del calor interior y la refrigeración natural. 
Las altísimas temperaturas que provoca la radiación solar por un acceso indiscriminado de energía a través de la cubierta produce graves problemas de confort térmico interior. No hay en toda la región necesidad de protección de los edificios de las bajas temperaturas.

El objetivo de la aplicación de los recursos o residuos de biomasa en la costa será el conseguir evitar la entrada de calor cuando, en ausencia de viento las temperaturas superen los $27^{\circ} \mathrm{C}$ así como la incidencia de la radiación solar sobre la envolvente del edificio.

El aislamiento de la cubiertas cobra por lo tanto en la costa importancia capital siendo posible rehabilitar las cubiertas de chapa o fibrocemento mediante un trasdosado que forme una cámara de aire ventilada entre la cubierta y el panel aglomerado de fibras naturales. Biomasa como el bagazo de caña de azúcar pueden conformarse fácilmente en este tipo de cielos rasos aislantes pudiendo ser revestidos posteriormente de tierra o yeso para aumentar su durabilidad. El trasdosado interior de las paredes de bloque de hormigón con dicha técnica podría convertirse en una rehabilitación energética, evolución aislante del tradicional enquinchado de caña.

Por otro lado, existen en la zona gran número de tipos de biomasa aprovechables para aislantes en la formación de bloques aglomerados con tierra y cal o cemento tales como los residuos de guadua, las fibras de coco o de palma, el bagazo, el raquis del banano, la cascarilla de arroz, la tuza del maíz y las cascarillas de café o cacao. Pese a ello, este tipo de solución constructiva basada en bloques macizos, al aportar a la edificación capacidad de acumulación de energía (inercia térmica), únicamente será útil en zonas de interior o el borde de la cordillera, en las cuales el descenso de la temperatura nocturna permita la ventilación y el enfriamiento de la casa. El aislamiento térmico evitará el acceso del calor al interior mientras el frio acumulado en la masa de los bloques durante la noche mantendrá fresco el interior.

La biomasa potencialmente más adecuada para el compuesto tierra + biomasa es aquella larga y resistente a esfuerzos de tracción, con un cierto grosor de forma que exista en las fibras cierta cantidad de aire encapsulado como ocurre con el bagazo de caña de azúcar, el raquis de banano o los residuos de guadua. Las fibras cortas como el cacao, la cascarilla de arroz o la tuza de maíz serán más adecuadas para compuestos de cemento debido a la mayor capacidad aglomerante de este. Los residuos fibrosos como el ráquis de palma o las fibras de coco, así como cualquiera de las biomasas anteriores convenientemente citadas, convenientemente preservadas (con sales de bórax o mineralización con cemento y agua) dotarán a cualquier cerramiento de un carácter aislante. Los sistemas tradicionales de madera de la región permitirían de una forma sencilla la transformación de este modo de las paredes en muros aislantes.

El bahareque o muros de entramado de palos, cañas o guadua revestido de barro o mortero de cemento ha sido y continúa siendo un sistema constructivo muy extendido en toda América Latina. El bahareque presenta interesantes características dado su bajo coste, su estructura antisísmica y 
su ligereza, pero una gran desventaja frente a otros tipos de muro tradicional: su limitadísimo aislamiento térmico debido, no tanto a su coeficiente de conductividad de la biomasa, si no al reducido espesor del propio sistema constructivo. Desde la presente investigación se vienen realizando pruebas para la utilización del bagazo de caña de azucar como estructura tejida o superpuesta como base aislante para recibir el revestimiento interior y exterior de tierra 0 cemento. Con ello se obtendría un tipo de bahareque aislante que mejoraría en zonas frías o cálidas de alta radiación la habitabilidad interior de la vivienda con materiales de deshecho como materia prima básica. Los principales retos a superar en dicha técnica constructiva innovadora son el ataque de microorganismos o insectos así como la formación de una subestructura lo suficientemente rígida como para conformar muros de altura suficiente (Velasco, Goyos, 2015). La Tabla 3 resume las biomasas disponibles en la costa así como los posibles usos aislantes.

Tabla 3. Biomasas y usos aislantes disponibles en la costa

\begin{tabular}{|c|c|c|c|c|c|c|c|c|c|c|c|c|c|c|c|c|c|}
\hline \multicolumn{18}{|c|}{ Distribución regional de los principales fibras de la costa y posibles usos aislantes } \\
\hline \multirow[b]{2}{*}{ Nombre común } & \multirow[b]{2}{*}{$\begin{array}{l}\text { Nombre } \\
\text { científico }\end{array}$} & \multicolumn{10}{|c|}{ Presencia en Provincia } & \multicolumn{6}{|c|}{ Posibles usos aislantes } \\
\hline & & 否 & 离 & $\begin{array}{l}\text { 종 } \\
\text { 좀 } \\
\text { 임 }\end{array}$ & 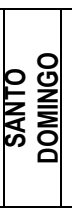 & 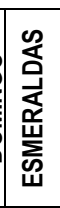 & 永 & 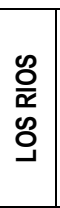 & 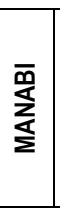 & 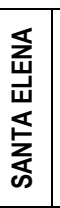 & $\begin{array}{l}\text { 옹 } \\
\text { ய }\end{array}$ & 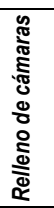 & 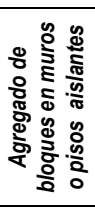 & 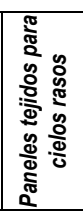 & 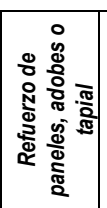 & 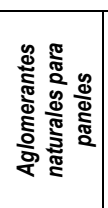 & 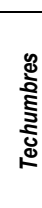 \\
\hline \multicolumn{18}{|l|}{ LEÑOSOS } \\
\hline Guadua & $\begin{array}{c}\text { Guadua } \\
\text { angustifolia }\end{array}$ & & & $\mathrm{x}$ & $\mathrm{x}$ & $\mathrm{x}$ & $\mathrm{x}$ & & & $\mathbf{X}$ & $\mathrm{x}$ & $\mathrm{x}$ & $\mathrm{X}$ & $\mathrm{x}$ & & & \\
\hline Caña & Arundo donax & & & $\mathbf{X}$ & & $\mathbf{X}$ & & & & & $X$ & $X$ & $X$ & $X$ & & & \\
\hline Caucho & $\begin{array}{c}\text { Ficus elástica } \\
\text { Hevea Brasiliensis }\end{array}$ & $\mathrm{X}$ & & $\mathrm{x}$ & $\mathbf{x}$ & $\mathrm{x}$ & & $\mathrm{x}$ & & & & & & & & $\mathrm{x}$ & \\
\hline Matapalo & $\begin{array}{c}\text { Ficus } \\
\text { nymphaeifollia }\end{array}$ & & & & & & $\mathrm{x}$ & & & & & & & & $\mathbf{x}$ & $\mathrm{x}$ & \\
\hline Balsa & $\begin{array}{c}\text { Ochroma } \\
\text { pyramidale }\end{array}$ & & & $x$ & & & $\mathrm{x}$ & $\mathrm{X}$ & & & $x$ & & $x$ & & & & \\
\hline Ceibo & $\begin{array}{l}\text { Erythrina } \\
\text { cristagalli }\end{array}$ & & & & & & & & $x$ & & & & $x$ & & & & \\
\hline \multicolumn{18}{|l|}{ MIMBRES } \\
\hline Totora & $\begin{array}{l}\text { Schoenoplectus } \\
\text { californicus }\end{array}$ & & $x$ & $\mathrm{x}$ & & & & & & & & $x$ & $x$ & $x$ & & & $\mathrm{x}$ \\
\hline Junco & $\begin{array}{c}\text { Typha } \\
\text { dominguensis }\end{array}$ & & & & & & & & $x$ & & & $\mathrm{x}$ & $x$ & $x$ & & & \\
\hline Totorilla & Juncus arcticus & & $X$ & $X$ & & & & & & & & $\mathbf{X}$ & $X$ & $\mathrm{X}$ & & & \\
\hline Pajonal & $\begin{array}{l}\text { Diversas } \\
\text { especies }\end{array}$ & $x$ & $x$ & $x$ & & & & & & & & & & & & & $\mathrm{x}$ \\
\hline Sigse & $\begin{array}{l}\text { Cortaderia nitida } \\
\text { (kunth) Pilg. }\end{array}$ & $x$ & $x$ & $x$ & & & & & & & $x$ & & & & $x$ & & $\mathrm{x}$ \\
\hline \multicolumn{18}{|l|}{ TEXTILES } \\
\hline Abacá & Musa textilis & & & $\mathrm{X}$ & $\mathrm{X}$ & $\mathrm{X}$ & & & $\mathrm{X}$ & & & & & & $\mathrm{X}$ & & \\
\hline Cabuya & Agave sisalana & $x$ & $x$ & $x$ & & & $x$ & & $x$ & & & & & & $\mathrm{x}$ & & \\
\hline Penco & Agave americana & $x$ & $\mathrm{x}$ & $\mathrm{x}$ & & & $x$ & & $x$ & & & & & & $\mathrm{x}$ & & \\
\hline Fique & $\begin{array}{c}\text { Furcraea } \\
\text { bedinghausii }\end{array}$ & $x$ & $x$ & $x$ & & & $x$ & & $x$ & & & & & & $x$ & & \\
\hline \multicolumn{18}{|l|}{ PALMERAS } \\
\hline Palma africana & Elaeis guineensis & & & $x$ & $x$ & $x$ & $x$ & $x$ & $x$ & & & $x$ & $x$ & & & & $x$ \\
\hline Toquilla & $\begin{array}{l}\text { Carludovica } \\
\text { palmata }\end{array}$ & & & & & & & & $x$ & & & & & & $x$ & & $x$ \\
\hline Coco & Cocos nucifera & & & & & $x$ & $x$ & & $x$ & & & $x$ & & & $x$ & & $\mathrm{x}$ \\
\hline Mocora & $\begin{array}{l}\text { Astrocaryum } \\
\text { standleyanum }\end{array}$ & & & & & $\mathrm{x}$ & & & $x$ & & & & & & $\mathrm{X}$ & & $\mathrm{x}$ \\
\hline
\end{tabular}




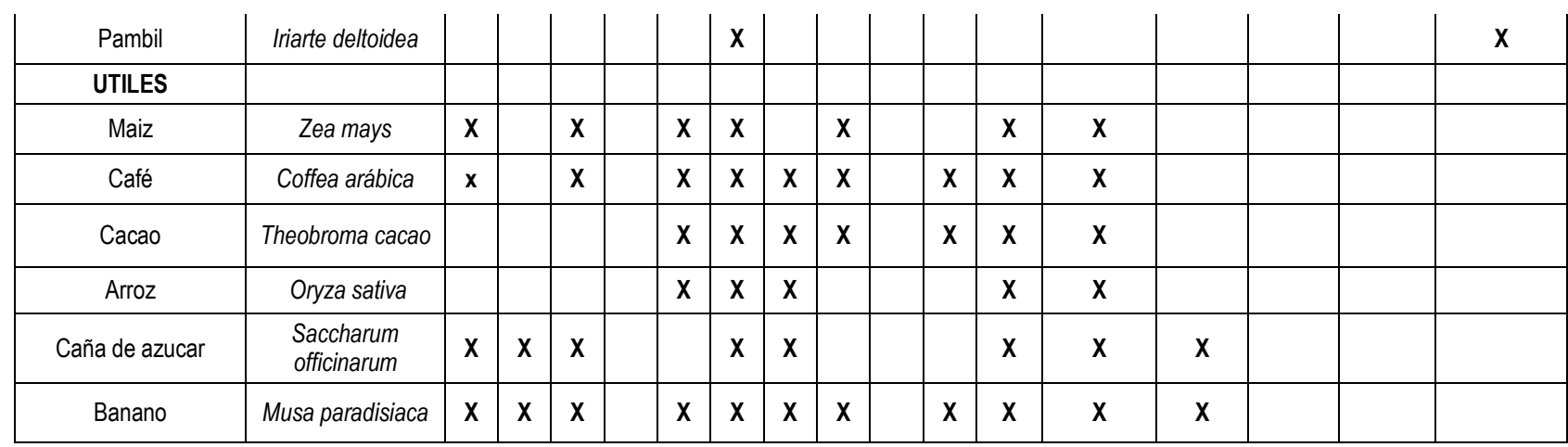

\subsubsection{Sierra}

La región de la Sierra está atravesada por la cordillera de los Andes que recorre el país de Norte a Sur. Comprende las provincias de Azuay, Bolívar, Cañar, Carchi, Chimborazo, Cotopaxi, Imbabura, Loja, Pichincha, Santo Domingo de los Tsáchilas y Tungurahua. Se divide en dos sistemas paralelos: Cordillera Oriental y Occidental, separados por llanuras y valles, creando una serie de microclimas conocidos como pisos o escalones climáticos (INAMHI, n.d.)

En el clima tropical andino la temperatura media varía entre las 20 y $25^{\circ} \mathrm{C}$. Las lluvias son escasas y la atmósfera seca. Comprende todas las tierras bajas hasta una altura de $1500 \mathrm{~m}$. El subtropical andino va desde los 1500 hasta los $2500 \mathrm{~m}$, con una temperatura media de $20{ }^{\circ} \mathrm{C}$. Lluvias abundantes en Invierno y poco frecuentes en verano lo caracterizan. El clima templado, con una temperatura media de $17^{\circ} \mathrm{C}$, se sitúa en los lugares que van desde los 2500 hasta los $3500 \mathrm{~m}$ de altitud. Se caracteriza por tener lluvias abundantes, granizadas frecuentes y ambiente nublado. Es la franja climática más poblada de la Sierra. El piso frío comprende todos aquellos lugares que van desde los 3500 hasta las $5650 \mathrm{~m}$. Su temperatura varia entre 1 y $10^{\circ} \mathrm{C}$ durante el día durante todo el año. Se dan lluvias torrenciales, neblinas espesas y lloviznas casi constantes. El clima glacial se sitúa por encima de los 5650 . Se caracteriza por registrar temperaturas Inferiores a los $0{ }^{\circ} \mathrm{C}$ y nieve perpetua. No es de interés al no estar habitado.

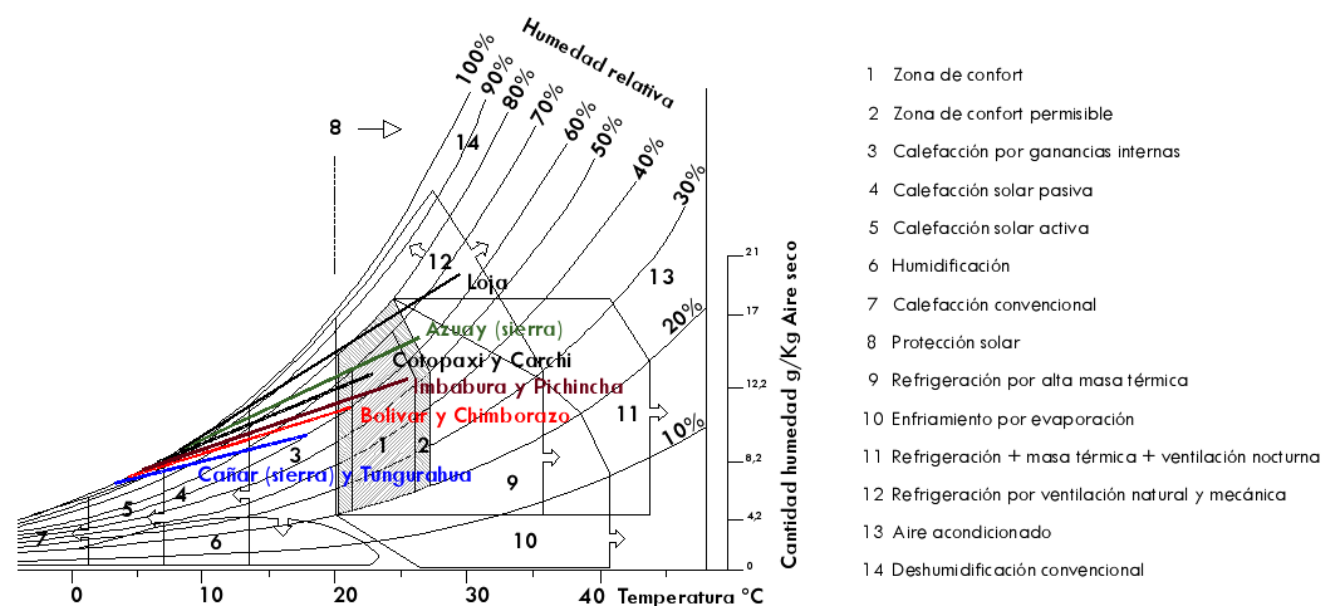

Figura 4. Gráfico bioclimático. Provincias de la región climática de sierra.

La Figura 4 nos muestra como en toda la región las bajas temperaturas exigen la presencia de 
aislamiento térmico e inercia térmica junto con algún tipo de sistema de calefacción. Pese a ello, la situación en la sierra es más compleja de lo que puede mostrar éste debido a la gran variabilidad de microclimas existente, por lo que los mapas de temperaturas mínimas nos ofrecen un instrumento clave para determinar la importancia de la utilización de la biomasa con fines aislantes para la protección de la envolvente en la región. Por el contrario, no existen en la región climática de la sierra problemas de altas temperaturas. Si pueden existir en cambio problemas puntuales derivados de la alta radiación en zonas de baja altitud en donde se hará necesario el control de la radiación solar.

Las edificaciones tradicionales en su mayor parte se caracterizan por muros de piedra o tierra (tapial o adobe), pisos de tierra y cubiertas de paja o teja sobre estructura de madera. En zonas menos frías se utiliza con frecuencia el bahareque que consiste en muros de cerramiento formados por una estructura de madera o cañas recubierta de barro y paja.

La construcción en tierra es un recurso constructivo de bajo impacto ambiental, acceso local e integrado en las tradiciones constructivas locales. Presenta inconvenientes de durabilidad si existe un exceso de humedad provocada por capilaridad del suelo o agua de lluvia y, más allá del efecto beneficioso de estabilidad térmica que produce en el interior de las construcciones, es un material con un alto coeficiente de conductividad por lo que resulta prácticamente imposible alcanzar las temperaturas de confort interior sin altísimos consumos energéticos.

En las zonas altas de los páramos se mantiene un tipo de choza mixta de muros de tierra y tejado de paja que se extiende prácticamente hasta el suelo. La gran capacidad aislante de la paja protege de los vientos fríos y el agua los muros de tierra. En todos los tipos constructivos los suelos apisonados constituyen el principal foco frío.

Pero un modelo único se extiende invariablemente en al actualidad por el país basado en muros de bloques de hormigón y techos de chapa o fibrocemento empeorando las condiciones interiores de habitabilidad frente al los tipos tradicionales. En las construcciones de techos de chapa o fibrocemento a las pérdidas de energía a través de muros y suelos conductivas se suman las grandes pérdidas energéticas producidas por la radiación espacial durante la noche, agravando los problemas de confort ya existentes en las tipologías tradicionales. Todo ello hace que este tipo de viviendas sean frecuentemente abandonadas o utilizadas como corrales.

El potencial de la inclusión de fibras en los compuestos de tierra está ya interiorizado en las distintas técnicas del adobe o del tapial con el objetivo de reforzar estructuralmente este. Estudios recientes elaborados por los autores (Velasco, Goyos, 2015), demuestran que es posible la mejora aislante de estas técnicas tradicionales mediante la adición de considerables cantidades de totora, cacao, café o pajonal sin una merma excesiva de la resistencia del compuesto. 
En zonas frías como las del páramo ecuatoriano la temperatura de los pisos en contacto con el terreno puede alcanzar temperaturas inferiores a $5^{\circ} \mathrm{C}$ de forma permanente, por lo que el aislamiento térmico de este elemento es imprescindible para alcanzar el confort térmico. Las solicitaciones requeridas al material base del piso se limitan a la resistencia a la abrasión de la capa superficial. Es por ello que el compuesto de tierra apisonada o estabilizada con reducidas cantidades de cal o cemento y altos porcentajes de biomasa atesora un enorme potencial como aislante de bajo coste. El campo de investigación en pisos aislantes se abre a prácticamente todo tipo de biomasa de la región.

Otra posible aplicación y dado el poder aislante demostrado de fibras como el pajonal es el desarrollo de sistemas constructivos que permitan confinar y preservar la biomasa, interponiéndola entre el interior y el exterior de una forma similar a las chozas actuales, pero protegidas de la intemperie. En zonas altas de la sierra, por ejemplo, en donde la presencia de pajonal es abundante y la existencia de insectos que puedan alimentarse de esta muy limitado, sería posible el uso de ésta, de forma local y sustentable, para el relleno de bloques de hormigón siempre y cuando se proteja la biomasas de la humedad del suelo o los muros de la incidencia agua de lluvia. En zonas en las que abunde la madera será posible abrir un nuevo campo de estudio consistente en confinar en cámaras dicha biomasa obteniéndose un muro de madera aislante. El principal reto a salvar es la conservación de dicha biomasa frente al ataque de insectos y hongos. La Tabla 4 resume las biomasas disponibles en la región así como los posibles usos aislantes.

Tabla 4. Biomasas y usos aislantes disponibles en la sierra

\begin{tabular}{|c|c|c|c|c|c|c|c|c|c|c|c|c|c|c|c|c|c|}
\hline \multicolumn{18}{|c|}{ Distribución regional de los principales fibras de la sierra y posibles usos aislantes } \\
\hline \multirow[b]{2}{*}{ Nombre común } & \multirow[b]{2}{*}{$\begin{array}{l}\text { Nombre } \\
\text { científico }\end{array}$} & \multicolumn{10}{|c|}{ Presencia en Provincia } & \multicolumn{6}{|c|}{ Posibles usos aislantes } \\
\hline & & 丕 & 离 & 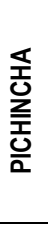 & 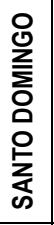 & $\begin{array}{l}\stackrel{\alpha}{\overleftarrow{\alpha}} \\
\underline{\underline{\partial}} \\
\text { ơ }\end{array}$ & 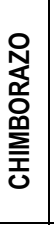 & $\begin{array}{l}\bar{x} \\
\text { à } \\
\text { o } \\
\text { ○ }\end{array}$ & 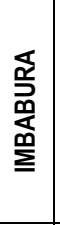 & 苃 & 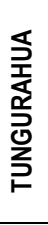 & 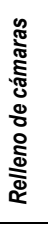 & 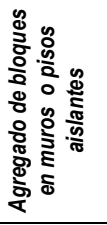 & 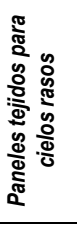 & 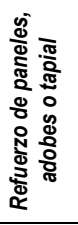 & 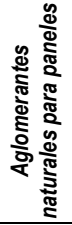 & 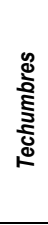 \\
\hline \multicolumn{18}{|l|}{ LEÑOSOS } \\
\hline Caña Guadúa & $\begin{array}{l}\text { Bambusa } \\
\text { guadua }\end{array}$ & & & $\mathbf{x}$ & $\mathrm{x}$ & & & & & & & $\mathrm{x}$ & $\mathrm{X}$ & & & & \\
\hline Guadua & $\begin{array}{c}\text { Guadua } \\
\text { angustifolia }\end{array}$ & & & $\mathbf{X}$ & $\mathrm{X}$ & & $\mathrm{x}$ & & & & & $\mathrm{x}$ & $\mathbf{X}$ & $\mathrm{X}$ & & & \\
\hline Caña & Arundo donax & & & $\mathbf{X}$ & & & & & & & & $\mathbf{X}$ & $\mathbf{X}$ & $\mathbf{X}$ & & & \\
\hline Caucho & $\begin{array}{c}\text { Ficus elastica } \\
\text { Hevea } \\
\text { Brasiliensis }\end{array}$ & $x$ & & $x$ & $x$ & & & & & & $x$ & & & & & $x$ & \\
\hline Balsa & $\begin{array}{c}\text { Ochroma } \\
\text { pyramidale }\end{array}$ & & & $x$ & & & & & & & & & $\mathrm{x}$ & & & & \\
\hline Ceibo & $\begin{array}{l}\text { Erythrina } \\
\text { cristagallii }\end{array}$ & & & & & & & & & $x$ & & & $x$ & & & & \\
\hline \multicolumn{18}{|l|}{ MIMBRES } \\
\hline Totora & $\begin{array}{l}\text { Schoenoplectus } \\
\text { californicus }\end{array}$ & & $\mathbf{x}$ & $\mathbf{x}$ & & & $x$ & $x$ & $x$ & $x$ & $x$ & $\mathbf{x}$ & $\mathbf{x}$ & $\mathbf{x}$ & & & $\mathbf{x}$ \\
\hline Totorilla & Juncus arcticus & & $\mathbf{X}$ & $\mathbf{X}$ & & & $\mathbf{X}$ & $\mathbf{X}$ & $\mathbf{X}$ & $\mathbf{X}$ & $\mathbf{X}$ & $\mathbf{X}$ & $\mathbf{X}$ & $\mathbf{X}$ & & & \\
\hline
\end{tabular}




\begin{tabular}{|c|c|c|c|c|c|c|c|c|c|c|c|c|c|c|c|c|}
\hline Pajonal & $\begin{array}{l}\text { Diversas } \\
\text { especies }\end{array}$ & $\mathrm{X}$ & $\mathrm{X}$ & $\mathrm{X}$ & $\mathrm{X}$ & $\mathrm{x}$ & $\mathrm{X}$ & $\mathrm{X}$ & $\mathrm{X}$ & $x$ & $x$ & & & & & $x$ \\
\hline Sigse & $\begin{array}{c}\text { Cortaderia nitida } \\
\text { (kunth) Pilg. }\end{array}$ & $x$ & $\mathrm{X}$ & $x$ & $\mathrm{X}$ & $\mathrm{x}$ & $\mathrm{x}$ & $\mathrm{X}$ & $\mathrm{x}$ & $\mathrm{X}$ & $\mathrm{x}$ & & & & $\mathrm{x}$ & $x$ \\
\hline \multicolumn{17}{|l|}{ TEXTILES } \\
\hline Abacá & Musa textilis & & & $x$ & $x$ & & & & & & & & & & $\mathrm{x}$ & \\
\hline Cabuya & Agave sisalana & $\mathrm{x}$ & $\mathrm{X}$ & $\mathrm{x}$ & & & $\mathrm{X}$ & & $\mathrm{x}$ & $\mathrm{x}$ & $\mathrm{X}$ & & & & $\mathrm{x}$ & \\
\hline Penco & $\begin{array}{c}\text { Agave } \\
\text { americana }\end{array}$ & $\mathrm{x}$ & $x$ & $\mathrm{x}$ & & & $x$ & & $x$ & $x$ & $x$ & & & & $x$ & \\
\hline Fique & $\begin{array}{c}\text { Furcraea } \\
\text { bedinghausii }\end{array}$ & $\mathrm{x}$ & $\mathrm{X}$ & $\mathrm{x}$ & & & $\mathrm{x}$ & & $x$ & $\mathrm{X}$ & $x$ & & & & $x$ & \\
\hline \multicolumn{17}{|l|}{ PALMERAS } \\
\hline Palma africana & $\begin{array}{c}\text { Elaeis } \\
\text { guineensis }\end{array}$ & & & $\mathrm{x}$ & $x$ & $\mathrm{x}$ & & $x$ & & & & $\mathrm{X}$ & $x$ & & & $x$ \\
\hline Coco & Cocos nucifera & & & & & & & & & $\mathrm{x}$ & & $x$ & & & $\mathrm{X}$ & $x$ \\
\hline Pambil & Iriarte deltoidea & & & & & & & & & & & & & & & $\mathrm{x}$ \\
\hline \multicolumn{17}{|l|}{ UTILES } \\
\hline Maiz & Zea mays & $x$ & & $x$ & & $x$ & $x$ & & $x$ & $\mathrm{x}$ & $x$ & $x$ & $x$ & & & \\
\hline Café & Coffea arábica & $\mathrm{X}$ & & $x$ & & & & $\mathrm{X}$ & & $\mathrm{X}$ & & $\mathrm{x}$ & $\mathrm{X}$ & & & \\
\hline Caña de azucar & $\begin{array}{l}\text { Saccharum } \\
\text { officinarum }\end{array}$ & $x$ & $x$ & $\mathrm{x}$ & & & $x$ & $x$ & $x$ & $x$ & & $x$ & $x$ & $x$ & & \\
\hline Banano & $\begin{array}{c}\text { Musa } \\
\text { paradisiaca }\end{array}$ & $x$ & $x$ & $x$ & & $x$ & & $x$ & & $x$ & & 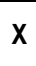 & $\mathrm{X}$ & $x$ & & \\
\hline
\end{tabular}

\subsubsection{Amazonia}

Esta región climática corresponde a las provincias de Morona Santiago, Napo, Orellana, Pastaza, Sucumbios y Zamora Chinchipe. En ella coexisten el clima Amazónico Húmedo y el Amazónico Semi-Húmedo, similares al que se produce en la parte interna de la región costera. La temperatura media varía entre 22 y $26^{\circ} \mathrm{C}$ y es la región más húmeda de Ecuador. Las precipitaciones son muy abundantes y frecuentes todo el año (más de $3000 \mathrm{~mm}$ anuales). La parte más cercana a la cordillera de los Andes es una zona densamente nublada, debido a que allí se condensan grandes masas de vapor proveniente del Atlántico y de la selva amazónica (Yepez, 2012).
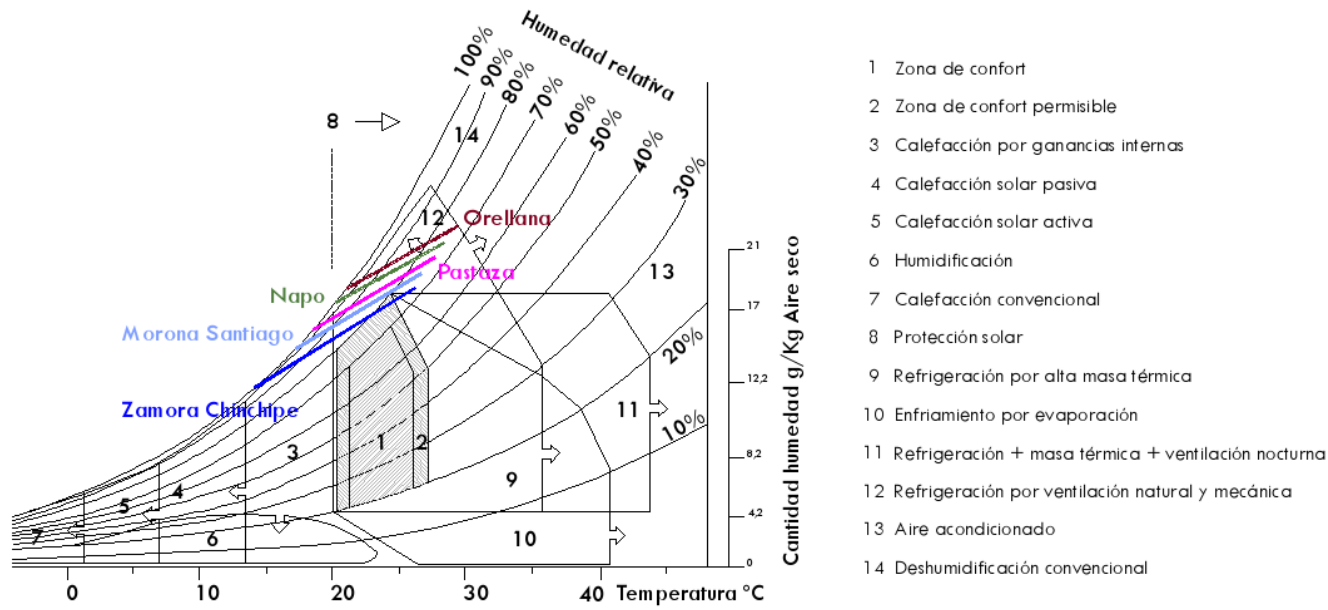

Figura 5. Gráfico bioclimático. Provincias de la región climática de la Amazonia.

Pese a las distintas etnias que las habitan, la similitud de recursos naturales y condicionantes 
climáticos hace que la arquitectura vernácula de esta regiones sea muy similar a la de la costa en cuanto a materiales y técnicas constructivas tradicionales. Los principales factores a considerar son la humedad y en menor grado las altas temperaturas. La edificación por lo tanto emplea materiales vegetales locales, facilitando la permeabilidad de toda la envolvente (llegando incluso en ocasiones a prescindir de las paredes) para evitar problemas de confort derivados de la humedad elevada. La protección solar de la cubierta se basa en una superposición de capas vegetales que forman sucesivas cámaras de aire ventiladas. Al igual que en todo el país la sustitución de los tipos constructivos tradicionales por sistemas constructivos de hormigón o cubiertas de fibrocemento o chapa de acero supone un empeoramiento radical de la sostenibilidad edificatoria y del confort al aumentar las temperaturas debido a la radiación solar y a la acumulación de calor en la inercia térmica de los materiales pétreos, los cuales dificultan enormemente la ventilación y refrigeración interior. La Tabla 5 resume las biomasas disponibles en la región así como los posibles usos aislantes.

Tabla 5. Biomasas y usos aislantes disponibles en la Amazonía

\begin{tabular}{|c|c|c|c|c|c|c|c|c|c|c|c|c|c|}
\hline \multicolumn{14}{|c|}{ Distribución regional de los principales fibras de la amazonia y posibles usos aislantes } \\
\hline \multirow[b]{2}{*}{ Nombre común } & \multirow[b]{2}{*}{ Nombre científico } & \multicolumn{6}{|c|}{ Presencia en Provincia } & \multicolumn{6}{|c|}{ Posibles usos aislantes } \\
\hline & & 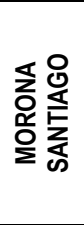 & $\frac{0}{\frac{1}{2}}$ & 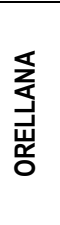 & 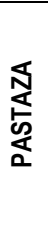 & 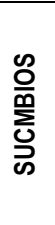 & 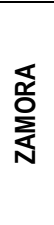 & 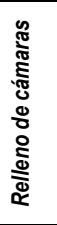 & 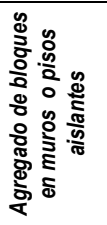 & 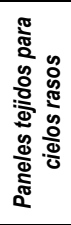 & 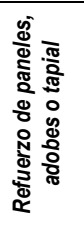 & 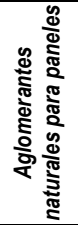 & 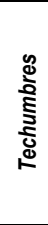 \\
\hline \multicolumn{14}{|l|}{ LEÑOSOS } \\
\hline Guadua & Guadua angustifolia & & $\mathrm{x}$ & & & $\mathrm{x}$ & & $\mathbf{x}$ & $\mathrm{X}$ & $\mathbf{x}$ & & & \\
\hline Caña Guadua & Arundo donax & & $\mathbf{X}$ & $\mathbf{X}$ & $\mathbf{X}$ & $\mathbf{X}$ & & $\mathbf{X}$ & $\mathbf{X}$ & $\mathbf{X}$ & & & \\
\hline Caucho & $\begin{array}{c}\text { Ficus elastica } \\
\text { Hevea Brasiliensis }\end{array}$ & $\mathrm{x}$ & $\mathrm{x}$ & & $\mathrm{x}$ & $\mathrm{x}$ & & & & & & $\mathbf{x}$ & \\
\hline Matapalo & Ficus nymphaeifollia & $\mathrm{x}$ & & & & & & & & & $x$ & $x$ & \\
\hline Ceibo & Erythrina cristagalli & & & & & & & & $x$ & & & & \\
\hline Bejuco real & Cissus verticillata & $\mathrm{x}$ & $x$ & & $x$ & & & & & & $x$ & & \\
\hline \multicolumn{14}{|l|}{ MIMBRES } \\
\hline Totora & Schoenoplectus californicus & & $x$ & & & & & $x$ & $\mathrm{x}$ & $\mathrm{x}$ & & & \\
\hline Totorilla & Juncus arcticus & & $\mathrm{x}$ & & & & & $\mathrm{x}$ & $x$ & $\mathrm{x}$ & & & \\
\hline Sigse & $\begin{array}{c}\text { Cortaderia nitida (kunth) } \\
\text { Pilg. }\end{array}$ & & & & & $x$ & & & & & $\mathrm{x}$ & & \\
\hline \multicolumn{14}{|l|}{ PALMERAS } \\
\hline Palma africana & Elaeis guineensis & & & $\mathrm{x}$ & & $\mathrm{x}$ & & $x$ & $\mathrm{x}$ & & & & $x$ \\
\hline Pambil & Iriarte deltoidea & & $\mathrm{x}$ & & $x$ & & & & & & & & $x$ \\
\hline \multicolumn{14}{|l|}{ UTILES } \\
\hline Maiz & Zea mays & & & & $x$ & & & $\mathrm{x}$ & $\mathrm{x}$ & & & & \\
\hline Café & Coffea arábica & & & & $x$ & & & $x$ & $x$ & & & & \\
\hline Cacao & Theobroma cacao & & $x$ & & $x$ & & & $x$ & $x$ & & & & \\
\hline
\end{tabular}

La sostenibilidad de las soluciones industrializadas se ve agravada por la distancia y dificultad de acceso a las zonas pobladas por lo que un retorno a patrones tradicionales convenientemente 
revisados es la zona amazónica de especial importancia. La aglomeración de fibras vegetales en forma de paneles (de cubierta o techo) será en este caso de principal interés y la preservación de la biomasa el factor clave del éxito, pues la pudrición y la presencia de hongos e insectos es muy importante en la zona.

No son recomendables la aglomeración de bloques o la mejora de los tipos constructivos de hormigón debidos a la acumulación de calor que ello supondría a excepción de las zonas más frías de la región de Morona Santiago o Zamora Chinchipe.

\section{Conclusiones}

La biomasa como componente aislante de composites o técnicas constructivas mixtas es útil en amplias zonas de Ecuador, tanto en zonas frías como en zonas cálidas. La utilización por lo tanto de biomasas incorporadas a los sistemas constructivos tradicionales tiene un gran potencial de mejora del hábitat en gran parte del país.

La diversidad agrícola y vegetal de Ecuador permite asegurar la existencia de biomasas vegetales aprovechables como componente aislante en la práctica totalidad del país habitado.

La integración de las soluciones innovadoras depende en gran medida de la aceptación de estas por parte de los usuarios y agentes implicados en la construcción. Es por ello que buscar sistemas que permitan una evolución de los sistemas tradicionales permite asegurar la implantación de las mejoras. La incorporación de biomasa local para el incremento del aislamiento es absolutamente compatible con los distintos sistemas constructivos de Ecuador. Así bloques, muros y adobes pueden continuar construyéndose de igual modo con idénticas técnicas. La incorporación de altos porcentajes de biomasas permitirá la mejora de dicha técnica. En ningún caso la sustitución por otra. En el caso de zonas cálidas en donde abundan las fibras vegetales largas que permiten conformar paneles, estos serán de gran utilidad para la mejora de las condiciones de hábitat con un incremento de coste muy limitado

Se demuestran por lo tanto las enormes posibilidades de uso de la biomasa en la mejora de las técnicas constructivas tradicionales de Ecuador en pos de la mejora de la eficiencia energética de las edificaciones.

\section{Bibliografía}

AENOR. (2008). UNE 41410 Bloques de tierra comprimida para muros y tabiques.

AEPEREACU. (n.d.). Clima de Ecuador.

Ali, M., Liu, H, A., Chouw, S. (2012) Construction And Building Materials, 30, 814-825. 
Alvarez-Castillo A., Salgado-Delgado, R. , García-Hernández, E., Domínguez-Domínguez, M.,

Beraldo A.L. (UPV). (2011). Aprovechamiento de residuos agro-industriales como fuente sostenible de materiales de construcción. CYTED.

Biagiotti, J., Puglia, D., Kenny J. M. (2004). Natural Fibers,2004,1

Borchsenius, F., (2006) In Botánica Económica De Los Andes Centrales, Ed. By B. Ø. M. Moraes R., L. P. Kvist, F. Borchsenius \& H. Balslev (2006) La Paz , Pp. 412-486.

Climate.data.org. (n.d.).

Cañadas, Luis. (1983). El Mapa Ecológico y Bioclimático de Ecuador.

David Nurnberg, Julio Estrada Ycaza, O. H. (1982). Arquitectura vernácula en el litoral.

de la Torre, H. N. L., Murielm P., Macía \& Balslev Editores, (2000) Enciclopedia de las plantas útiles del Ecuador

de la Torre, L. , Muriel, P., Balslev, H. (2006) Etnobotánica En Los Andes Del Ecuador

Dicker, M. P. M. Duckworth, P. F. Baker, A.B.. Francois G., Hazzard, M. K.. Weaver, P. M (2014) Composites: Part A 2014, 56 280-289.

Erriguren, J., (2013) Big Bamboo Alternativa Ecológica en la Construcción, , Vol. Sept-Oct

Franco P. J., Licea-Claveríe A. , Mendoza-Martínez A.M. (2012) Revista lberoamericana de Polímeros, 15,140-150.

Granandos-Baeza J.M., Aguirre-Cruz, A., Carmona-García, R. , Morales-Cepeda, A. , HerreraAndino P., (2001)Guia de Plantas Utiles de los Páramos de Zulueta, Ecuador

Hidalgo, A. (2007). Valoración de los residuos de biomasa en la industría de la Construcción. Situación de La Industria de La Construcción Y ..., 1-5.

Informe Técnico Parcial Del Proyecto: "Desperdicios Agrícolas e Industriales como Fuente Alternativa Para La Producción De Materiales Suplementarios en Concreto, CLAVE SIP20060647

INAMHI. (n.d.). Anuarios meteorológicos.

INEC (2009) ANÁLISIS DEL SISTEMA AGROALIMENTARIO DEL BANANO EN EL ECUADOR

INEC (2009),Sistema Agroalimentario Del Maíz 
INEC (2013) , In Book SISTEMA AGROALIMENTARIO DEL ARROZ

INEN. (1978). INEN 265. Ladrilos determinación de la resistencia a la flexión.

Izco, J.,Pulgar, I.,Aguirre, Z., Santin F., Rev. Peru. Biol. 2007, 14, 237-246.

Juárez, C., Guevara, B., Valdez, P. , Durán-Herrera, A., (2010) Construction And Building Materials 24, 1536-1541.

Katzer, J., Domski, J. (2013) Construction And Building Materials 2013, 38 790-795.

Pourrut, P., Rovere, O., Romo, I., \& Villacrés, H. (1995). Clima del Ecuador. El Agua En El Ecuador, 13-26.

Mantia, F. P. L., Morreale, M. (2011) Composites: Part A, 42 579-588.

Macía M.J. (2006) In Botánica Económica De Los Andes Centrales, Ed. By B. Ø. M. Moraes R., L.

P. Mena-Vásconez (2002) Congreso Mundial De Páramos Paipa, Colombia.

Romero, L.N, Weeks,S., Huelman, P., PLEA 2012 - 28th Conference, Opportunities, Limits \& Needs Towards An Environmentally Responsible Architecture, Lima, Perú, 2012.

Salazar, J., García, C. D., \& Olaya, J. M. (n.d.). Dosificación de hormigones ligeros con cascarilla de café. Ingeniería E Investigación, 51-56.

Salazar, S., Kerguelen, H., Cruz, J., Palacio M., (2007) Scientia Et Technica Año XIII, 13, 719723.

Serrano, J. S., \& Castro, J. V. (1985). Materiales de construcción con propiedades aislantes a base de cáscara de arroz. Informes de La Construcción, 37(372), 53-64.

Velasco, L., Delgado, R., \& Goyos, L. (2015). Investigación y desarrollo de aislantes térmicos naturales basados en residuos de biomasa para su aplicación en la mejora de la eficiencia energética de las edificaciones en América Latina

Velasco, L., Delgado, R., \& Goyos, L., Freire, (2015). Instalación para medición de conductividad térmica en composites basados en residuos de biomasa

Yepez, D. (2012). Análisis de la arquitectura vernácula del Ecuador: Propuestas de una arquitectura contemporánea sustentable. 